\title{
Peripheral Sensory Neuropathy
}

National Cancer Institute

\section{Source}

National Cancer Institute. Peripheral Sensory Neuropathy. NCI Thesaurus. Code C3501.

Inflammation or degeneration of the sensory nerves. 